Osteogenic impact of football training in 55- to 70-year-old women and men with prediabetes

Skoradal, May-Britt; Helge, Eva Wulff; Jørgensen, Niklas Rye; Mortensen, Jann; Weihe, Pál;

Krustrup, Peter; Mohr, Magni

Published in:

Scandinavian Journal of Medicine \& Science in Sports

DOI:

10.1111/sms.13252

Publication date:

2018

Document version

Publisher's PDF, also known as Version of record

Document license:

$\mathrm{CC} B \mathrm{BY}$

Citation for published version (APA):

Skoradal, M-B., Helge, E. W., Jørgensen, N. R., Mortensen, J., Weihe, P., Krustrup, P., \& Mohr, M. (2018).

Osteogenic impact of football training in 55- to 70-year-old women and men with prediabetes. Scandinavian Journal of Medicine \& Science in Sports, 28(Suppl. 1), 52-60. https://doi.org/10.1111/sms.13252 


\title{
Osteogenic impact of football training in 55- to 70-year-old women and men with prediabetes
}

\author{
May-Britt Skoradal $^{1} \quad$ | Eva Wulff Helge ${ }^{2}$ (i) ～Niklas R. Jørgensen ${ }^{3}$ (i) ～Jann Mortensen ${ }^{4,5}$ | \\ Pál Weihe $^{1,6} \mid$ Peter Krustrup $^{7,8}$ (D) $\mid$ Magni Mohr ${ }^{1,7,9}$
}

${ }^{1}$ Faculty of Health Sciences, Centre of Health Science, University of the Faroe Islands, Tórshavn, Faroe Islands

${ }^{2}$ Department of Nutrition, Exercise and Sports, University of Copenhagen, Copenhagen, Denmark

${ }^{3}$ Department of Clinical Biochemistry, Rigshospitalet, Copenhagen University Hospital, Glostrup, Denmark

${ }^{4}$ Department of Medicine, The Faroese National Hospital, Tórshavn, Faroe Islands

${ }^{5}$ Department of Clinical Physiology, Nuclear Medicine \& PET, Rigshospitalet, Copenhagen University Hospital,

Copenhagen, Denmark

${ }^{6}$ Department of Occupational Medicine and Public Health, The Faroese Hospital System, Tórshavn, Faroe Islands

${ }^{7}$ Department of Sports Science and Clinical Biomechanics, SDU Sport and Health Sciences Cluster (SHSC), Faculty of Health Sciences, University of Southern Denmark, Odense, Denmark

${ }^{8}$ Sport and Health Sciences, University of Exeter, Exeter, UK

${ }^{9}$ Center for Health and

Performance, Department of Food and Nutrition, and Sport Science, University of Gothenburg, Gothenburg, Sweden

Correspondence: Magni Mohr, Centre of Health Sciences, Faculty Health Sciences, University of the Faroe Islands, Jónas Broncksgøta 25, 3rd floor, Tórshavn, Faroe Islands (magnim@setur.fo).

Funding information

Faroese Research Council; Faroese Football Association; Faroese Diabetes Organisation
The effects of football training on bone health were examined in 55- to 70-year-old sedentary women and men with prediabetes. Patients $(n=50)$ with prediabetes (age; $61 \pm 9$ years, BMI $29.7 \pm 0.6 \mathrm{~kg} / \mathrm{m}^{2}$, body fat content; $37 \pm 1 \%, \mathrm{VO}_{2 \max }$; $22.7 \pm 0.8 \mathrm{~mL} / \mathrm{min} / \mathrm{kg}$ and mean arterial pressure; $104 \pm 3 \mathrm{~mm} \mathrm{Hg}$ ) were randomized into a football training group (FTG; $\mathrm{n}=27,14$ women) and a control group (CON; $\mathrm{n}=23,11$ women). At baseline, $73 \%$ and $24 \%$ were diagnosed with femur osteopenia and osteoporosis, respectively. FTG performed football training twice weekly 30-60-minute sessions in 16 weeks, and both FTG and CON received professional dietary advice. Pre- and post-intervention whole-body and regional bone mineral content (BMC) and density (BMD) were determined with DXA-scans, and venous blood samples were drawn and analyzed for plasma bone turnover markers. Change scores were greater $(P<0.05)$ in FTG compared to CON in leg BMD $\left(0.023 \pm 0.005\right.$ vs $\left.-0.004 \pm 0.001 \mathrm{~g} / \mathrm{cm}^{2}\right)$ and in leg BMC $(32 \pm 8 \mathrm{vs}-4 \pm 6 \mathrm{~g})$. Between-group changes in favor of FTG $(P<0.05)$ also occurred in the femur neck BMD (3.2\%) and femur shaft BMD (2.5\%). Whole-body BMC and BMD were unchanged in both groups during the intervention. In FTG, resting plasma osteocalcin, P1NP, and CTX-1 rose $(P<0.05)$ by $23 \pm 8,52 \pm 9$ and $38 \pm 7 \%$, with greater change scores $(P<0.05)$ than in CON. Finally, P1NP (formation)/CTX-1 (resorption) ratio increased $(P<0.05)$ in FTG $(127 \pm 15$ vs $150 \pm 11)$ from pre- to postintervention, with no change in CON $(124 \pm 12$ and $123 \pm 12)$. In conclusion, football training provides a powerful osteogenic stimulus and improves bone health in 55- to 70-year-old women and men diagnosed with prediabetes.

\section{K E Y W O R D S}

bone health, bone markers, bone mass, bone mineral density, osteocalcin, soccer

\section{1 | INTRODUCTION}

Bone health is deteriorating progressively with increasing age in both genders resulting in an elevated risk of developing osteoporosis and osteopenia. In the United States alone, 45 million meet the criteria for these two skeletal deficiencies. ${ }^{1}$ Especially in the post-menopausal period in women ${ }^{2}$ and at corresponding ages in men $^{3}$ bone health impairs rapidly. 
Impaired bone health combined with poor physical fitness may therefore increase the risk of falls and bone fractures markedly. In Europe, osteoporosis affected more than 22 million women aged $>50$ years or $22 \%$ of the female population in $2010 .{ }^{4}$ For men $>50$ years of age in the United States, it is estimated that around $25 \%$ will experience a fracture due to osteoporosis, ${ }^{1}$ and in 2050 , the incidence of hip fracture in men is expected to increase more than threefold worldwide. ${ }^{5}$ In both gender $>60$ years of age, fractured patients have a significantly higher mortality rate than the general population, and men suffering any major fracture display higher mortality rates than women. ${ }^{6}$ It seems that aging as well as a reduced amount of osteogenic activities with increasing age is responsible for the impaired bone health. Thus, maintaining a physically active lifestyle and performing regular exercise training with high osteogenic stimuli despite an old age may improve bone health and delay the onset of age-related osteoporosis and osteopenia. In geographical regions far north on the northern hemisphere, there is limited sunlight in the autumn and winter, which may affect the systemic D-vitamin levels and reinforce the age-induced deterioration in bone health. Therefore, the population in these regions may be at higher risk of poor bone health.

Physical inactivity is a global pandemic ${ }^{7}$ and is an independent risk factor for major chronic diseases. Type 2 diabetes mellitus (T2DM), prediabetes, and metabolic syndrome are common endocrine diseases in most parts of the world and are pathological phenomena closely associated with an inactive lifestyle. However, patients suffering from these metabolic deficiencies also have an elevated risk of other pathological conditions such as poor bone health. ${ }^{8}$ For example, various aspects of bone structure, density, skeletal integrity, as well as biochemical markers of bone turnover appear to be affected by poor glycemic control and remain a frequently overlooked complication of prediabetes and T2DM. ${ }^{9}$ Indeed, patients with deteriorated glycemic control are more susceptible to fractures than healthy controls, ${ }^{10,11}$ which appears to associate with impaired or poor glycemic control, longer disease duration, and complications of diabetes. ${ }^{8}$ Thus, it is of great importance to develop treatment and prevention protocols aiming to facilitate bone health in patients with prediabetes.

Weight-bearing exercise training is a well-known stimulator of osteogenesis and exercise-induced increases in bone mineralization. ${ }^{12}$ In addition, there appears to be consensus in the literature that bone deformation (strain) due to mechanical loading is required to stimulate osteogenesis. ${ }^{13}$ Moreover, the larger the strain magnitude and rate, as well as the magnitude of the variation between usual and novel strain, the larger the osteogenic gain. ${ }^{14}$ Zhao and Zhang (2014) ${ }^{15}$ reported meta-analysis data indicating marked benefits from high-impact exercise protocols on especially the lower limbs. Thus, large strain stimuli completed in few repetition cycles may be essential components of a training protocol aiming to promote osteogenesis and bone quality, ${ }^{13}$ and odd-impact as well as multimodal training regimens including resistance training, high intensity aerobic exercise, and balance training are recommended to improve bone strength and reduce the risk of falls and fractures. ${ }^{16,17}$

Recreational football training has substantial effect on bone turnover and increases in BMC or BMD have been reported in middle-aged ${ }^{18,19}$ and elderly men, ${ }^{20,21}$ as well as in women across the lifespan. ${ }^{19,22,23}$ For example, in a study of untrained premenopausal women, a 2\%-3\% improvement in tibia BMD measured with peripheral quantitative computed tomography (pQCT) was reported after 14 weeks of football training which was superior to continuous running. ${ }^{24}$ Finally, football training has been demonstrated to elicit a greater osteogenic stimulus compared to none-weight-bearing activities such as high and low intensity swimming. ${ }^{25}$ Thus, football training may represent efficient multimodal osteogenic training for a sedentary elderly patient group.

As it is reported that T2DM seems to impair bone strength independently of BMD due to an unfavorable change in bone turnover (Kulkarni 2017), ${ }^{8}$ it is highly warranted to test the hypothesis that football training induces a powerful osteogenic stimulus and causes site-specific osteogenic adaptations in sedentary, prediabetic 55- to 70-year-old women and men from the Faroe Islands. Especially for a country in the far northern hemisphere, knowledge about the role of osteogenic exercise in health promotion is demanded.

Please click on this video link to hear more about the study.

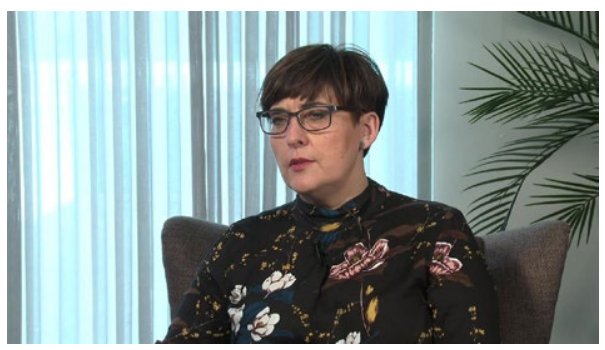

\section{$2 \mid$ METHODS}

\section{1 | Participants}

The participants in the present study were recruited from a population-based cross-sectional survey aiming to determine the prevalence of T2DM and prediabetes among the population aged 40-74 years in the Faroe Islands. The database included 1772 individuals, corresponding to $\sim 10 \%$ of the entire population aged $40-74$ years. ${ }^{26}$

A total of 117 individuals aged 55-70 years from the respective cohort were diagnosed with prediabetes $(\mathrm{HbA} 1 \mathrm{c} \geq 5.8 \%$, $40 \mathrm{mmol} / \mathrm{mol}$ ) and received a letter of invitation to participate in the study. The letter contained information about the study 
TA B L E 1 Participant characteristics at baseline $(\mathrm{n}=55)$

\begin{tabular}{lllll} 
Age $(\mathbf{y})$ & Height $(\mathbf{c m})$ & Weight $(\mathbf{k g})$ & BMI $\left(\mathbf{k g} / \mathbf{m}^{2}\right)$ & VO $_{2 \max }(\mathbf{m L} / \mathbf{m i n} / \mathbf{k g})$ \\
\hline $60 \pm 1$ & $170 \pm 1$ & $86.2 \pm 2.3$ & $29.7 \pm 0.6$ & $22.3 \pm 0.8$ \\
\hline SBP $(\mathbf{m m} \mathbf{H g})$ & DBP $(\mathbf{m m ~ H g})$ & MAP $(\mathbf{m m ~ H g})$ & RHR (beats/min) & Femoral neck T-score \\
\hline $142 \pm 3$ & $86 \pm 2$ & $104 \pm 2$ & $72 \pm 1$ & $-1.56 \pm 0.17$ \\
\hline
\end{tabular}

Age, body composition, maximal oxygen uptake, blood pressure, resting heart rate, and femoral neck T-score of the total participant sample recruited for the study. Data are means $\pm \mathrm{SE}$.

design and the experimental procedure that the participants would undergo. The letter was followed up by a phone call to clarify any questions and to invite the patients to an informational group meeting near their residence. The participant group is described in further detail in the paper by Skoradal et al. ${ }^{27}$ At baseline, $30 \%(n=15)$ of the participants had femoral osteoporosis (T-score $<-2.5)$ and 45\% $(\mathrm{n}=22)$ had femoral osteopenia (T-score $<-1.0$ ). Thus, $75 \%$ of the participants had either osteoporosis or osteopenia in femur (see Table 1). Additionally, spine osteoporosis and osteopenia were detected in numerous of the participants. The participants suffering from osteoporosis and osteopenia were informed on the increased risk of bone fractures prior to the intervention. After being informed verbally and in writing about the experimental procedures and associated risks, 55 individuals (28 men and 27 women; see Table 1) volunteered to participate in the study and gave their written consent. The study was approved by the ethical committee of the Faroe Islands and conducted in accordance with the Declaration of Helsinki (1964). Data from the study on the cardiovascular adaptations have been published in an accompanying manuscript by Skoradal et al. ${ }^{27}$

\section{2 | Experimental design}

The study applied a randomized controlled design (RCT). The participants reported to the laboratory and had a fasting blood sample taken pre- and post-intervention. Moreover, bone mineral content (BMC) and density (BMD) were assessed with pre- and post-DXA-scans. Descriptive participant data such as body fat content, height, weight, blood pressure, and maximal oxygen uptake were also obtained at baseline (see Table 1). Dietary advice was given to all participants by a registered dietitian in a 3-hour group-based session pre-intervention (see Ref. [27]). Subsequently, the participants were randomized to a football training group $(\mathrm{FTG} ; \mathrm{n}=32)$ and a control group $(\mathrm{CON} ; \mathrm{n}=23)$. Males and females were randomized separately to insure an equal gender distribution in the two groups. There were five drop-outs from FTG (three men and two women) due to low training attendance; $<1$ session per week, resulting in 27 participants that completed the FTG intervention and were included in the data analysis. There was no drop-out from CON $(n=23)$. There were examples of severe muscle soreness as result of the football training treatment in the initial phase of the intervention period, but no serious injuries occurred during the intervention period.

\section{3 | Resting blood sampling}

On the day of testing, participants reported to the laboratory after transport by car or bus. No training was performed 4872 hour prior to testing, and the participants were instructed to avoid physical activity on the day prior to the experimental testing. A blood sample was collected at rest pre- and postintervention under standardized conditions from an antecubital vein between 7 and 8 AM after an overnight fast using the venipuncture technique. The blood samples were rapidly centrifuged for 30 seconds, and the plasma was collected. The plasma samples were frozen at $-80^{\circ}$ and subsequently analyzed for bone turnover markers (BTM), that is, procollagen type $1 \mathrm{~N}$ propeptide (P1NP), osteocalcin, C-terminal telopeptide 1 (CTX-1), using ELISA and AlphaLISA apparatus (PerkinElmer, Cambridge, United Kingdom) at the scientific laboratory at the Research Center for Ageing and Osteoporosis, Departments of Diagnostics and Medicine, Copenhagen University Hospital Glostrup, Glostrup, Denmark.

\section{4 | Body composition}

Whole-body BMC and BMD were determined by total-body DXA-scanning (Norland XR-800; Norland Corporation, Oslo, Norway). The body was segmented in accordance with standard procedures to evaluate regional BMC and BMD, and all analyses were performed using Illuminatus DXA software (Norland Corporation, Oslo, Norway). The effective radiation dose was $<0.3 \mathrm{mSv}$ per whole-body scan. Additionally, sitespecific scans of the lumbar spine and proximal femur were conducted as previously described. ${ }^{25} \mathrm{BMD}$ in the lumbar vertebrae L2, L3, and L4, and in the femoral neck, -trochanter, and -shaft were evaluated. The radiation dose for these specific scans ranged between 3.0-4.5 mSv.

\section{5 | Training intervention}

The football training group completed a total of $32 \pm 2$ (range: 19-46) football training sessions over the 16 -week intervention 
period, corresponding to $2.0 \pm 0.1(1.2-2.9)$ weekly sessions. Each session lasted 30-60 minutes (30 minutes during weeks 0-2, 40 minutes during weeks 3-4, 50 minutes during weeks 5-6, and 60 minutes during weeks 7-16). The sessions were organized with two equally long periods $(2 \times 15$ minutes, $2 \times 20$ minutes, $2 \times 25$ minutes, $2 \times 30$ minutes progressively in the different weeks-see above) separated by 2-3 minutes of passive recovery. All sessions were organized as smallsided games (4v4-6v6), as previously described, ${ }^{28}$ and were preceded by a 10-minute warm-up period, as described by Uth et al. ${ }^{21}$ Heart rate was measured during one training session in week 4 and one session in week 12 of the intervention, and mean and peak heart rate were $127 \pm 5$ and $153 \pm 4 \mathrm{bpm}$, respectively, corresponding to $79 \pm 1$ and $96 \pm 1 \% \mathrm{HR}_{\max }$, respectively. All participants were given group-based dietary advice by a registered dietitian and provided with a standardized meal plan (see Skoradal et al for details). ${ }^{27}$

\section{6 | Statistical analysis}

Data are presented as means $\pm \mathrm{SE}$, unless otherwise stated. Statistical analyses were performed using SPSS v.22. After testing for normality, a two-factor mixed ANOVA was used to test the intervention-induced difference between FTG and CON for all plasma BTM, as well as BMC and BMD. When significant interactions or main effects were detected, data were subsequently analyzed using Bonferroni post-hoc t tests. The Pearson's product-moment method was used to test for associations in pre-to-post changes between selected variables. The significance level was $P<0.05$.

\section{3 | RESULTS}

\subsection{Bone mineral content and density}

Whole-body BMC and BMD at baseline were similar in FTG and CON, as well as in the measured site-specific skeletal regions (Table 2). During the intervention period, the change score in leg BMD was greater $(P<0.05)$ in FTG than in CON $\left(0.023 \pm 0.005\right.$ vs $-0.004 \pm 0.001 \mathrm{~g} / \mathrm{cm}^{2}$, Figure $\left.1 \mathrm{~A}\right)$. Leg BMC also increased more $(P<0.05)$ in FTG than in CON ( $32 \pm 8$ vs $-4 \pm 6 \mathrm{~g}$, Figure 1B). Evaluated by the wholebody scan, no significant changes occurred in any other major skeletal region (Table 2). The site-specific scans revealed between-group changes in BMD in the femoral neck and -shaft $(P<0.001)$ (Figure 2A). No significant difference was observed in the femoral trochanter BMD change score between FTG and CON $(P=0.09)$, whereas the change score in femoral trochanter BMC was greater $(P<0.05)$ in FTG than in $\operatorname{CON}(0.38 \pm 0.27$ vs $-0.31 \pm 0.12 \mathrm{~g}$; Figure $2 \mathrm{~A})$. Withingroup increases in BMC occurred in femoral trochanter $(P<0.05)$, while BMD increased in all three femoral sites and in vertebrae L2 and L3 in FTG only $(P<0.05$; Figure $2 \mathrm{~B})$. In contrast, a decrease $(P<0.05)$ in femur trochanter BMC and femur shaft BMD occurred in CON (Figures $2 \mathrm{~A}$ and $2 \mathrm{~B}$ ).
TA B L E 2 Whole-body and regional bone mineral content (BMC) and bone mineral density (BMD) before and after 16 wk of football training (FTG; $n=27$ ) and control (CON; $\mathrm{n}=23)$

\begin{tabular}{|c|c|c|c|c|}
\hline & \multicolumn{2}{|l|}{ FTG } & \multicolumn{2}{|l|}{$\mathrm{CON}$} \\
\hline & Pre & Post & Pre & Post \\
\hline \multicolumn{5}{|l|}{ Whole-body } \\
\hline $\mathrm{BMC}(\mathrm{g})$ & $2843 \pm 62$ & $2847 \pm 63$ & $2836 \pm 107$ & $2833 \pm 108$ \\
\hline $\operatorname{BMD}\left(\mathrm{g} / \mathrm{cm}^{2}\right)$ & $1.006 \pm 0.017$ & $1.007 \pm 0.016$ & $1.023 \pm 0.035$ & $1.027 \pm 0.034$ \\
\hline \multicolumn{5}{|l|}{ Head } \\
\hline $\mathrm{BMC}(\mathrm{g})$ & $457 \pm 14$ & $457 \pm 13$ & $451 \pm 23$ & $452 \pm 23$ \\
\hline $\operatorname{BMD}\left(\mathrm{g} / \mathrm{cm}^{2}\right)$ & $1.686 \pm 0.046$ & $1.723 \pm 0.046$ & $1.665 \pm 0.021$ & $1.673 \pm 0.027$ \\
\hline \multicolumn{5}{|l|}{ Chest } \\
\hline $\mathrm{BMC}(\mathrm{g})$ & $522 \pm 13$ & $515 \pm 14$ & $530 \pm 20$ & $517 \pm 19$ \\
\hline $\operatorname{BMD}\left(\mathrm{g} / \mathrm{cm}^{2}\right)$ & $0.896 \pm 0.022$ & $0.898 \pm 0.021$ & $0.907 \pm 0.043$ & $0.907 \pm 0.040$ \\
\hline \multicolumn{5}{|l|}{ Midriff } \\
\hline BMC (g) & $49 \pm 3$ & $44 \pm 3$ & $52 \pm 3$ & $49 \pm 3$ \\
\hline $\operatorname{BMD}\left(\mathrm{g} / \mathrm{cm}^{2}\right)$ & $1.379 \pm 0.041$ & $1.323 \pm 0.037$ & $1.358 \pm 0.067$ & $1.331 \pm 0.063$ \\
\hline \multicolumn{5}{|l|}{ Pelvis } \\
\hline BMC (g) & $354 \pm 11$ & $353 \pm 12$ & $340 \pm 16$ & $342 \pm 17$ \\
\hline $\operatorname{BMD}\left(\mathrm{g} / \mathrm{cm}^{2}\right)$ & $1.118 \pm 0.030$ & $1.108 \pm 0.028$ & $1.143 \pm 0.054$ & $1.133 \pm 0.052$ \\
\hline \multicolumn{5}{|l|}{ Arms } \\
\hline BMC (g) & $411 \pm 10$ & $426 \pm 11$ & $399 \pm 10$ & $413 \pm 11$ \\
\hline $\operatorname{BMD}\left(\mathrm{g} / \mathrm{cm}^{2}\right)$ & $0.666 \pm 0.017$ & $0.662 \pm 0.014$ & $0.689 \pm 0.016$ & $0.702 \pm 0.018$ \\
\hline
\end{tabular}

Data are presented as mean \pm SE. 

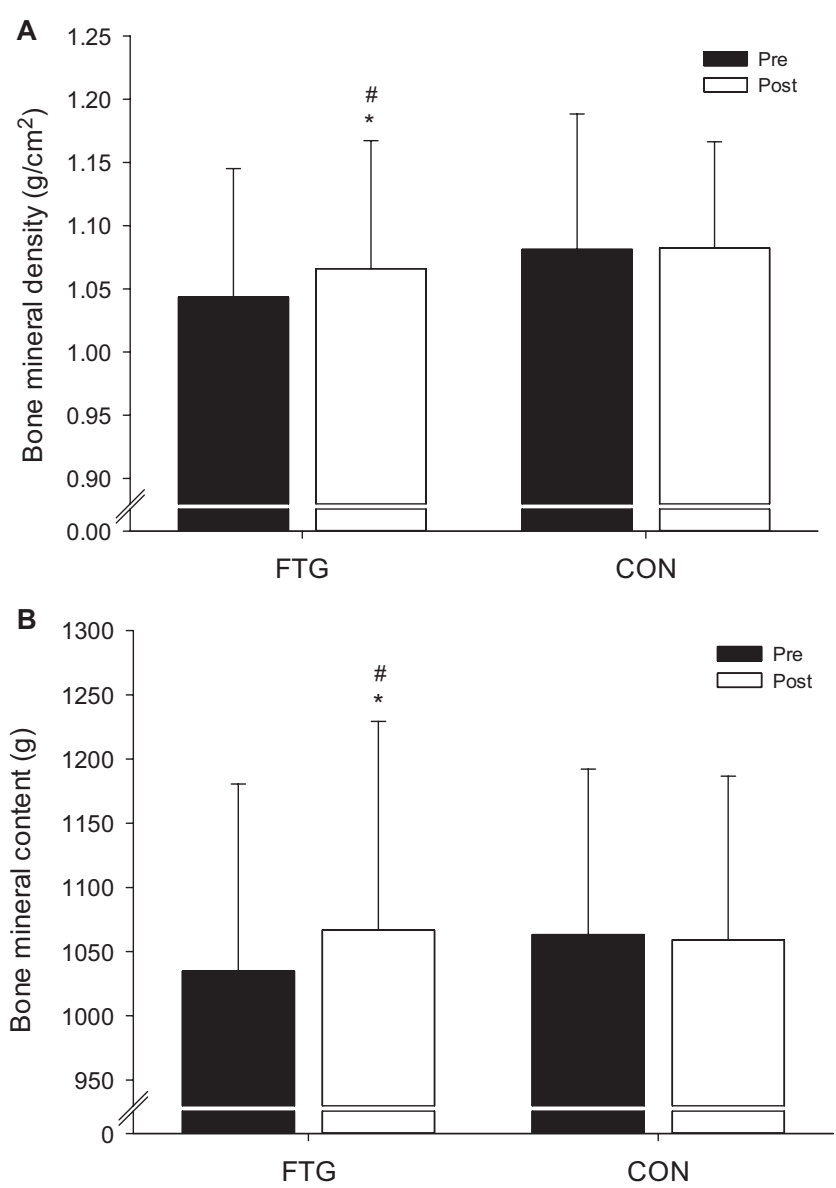

FIG URE 1 Leg bone mineral density (A) and content (B) in FTG and CON pre- and post-intervention. *Denotes a significant difference from pre. \#Denotes a significant difference in change score from CON. Significant level $P<0.05$. Data are means \pm SD (standard deviation)

\section{2 $\quad$ Bone turnover markers}

Baseline BTM did not differ between FTG and CON. In FTG, plasma osteocalcin, P1NP, and CTX-1 were $22.7 \pm 1.8$, $48.9 \pm 4.2$, and $0.47 \pm 0.05 \mathrm{ng} / \mathrm{mL}$ prior to training and increased $(P<0.05)$ during the intervention period by $23 \pm 8$, $52 \pm 9$, and $38 \pm 7 \%$, with no changes in CON (Figure 3). The change scores in FTG were greater $(P<0.05)$ than in CON (Figure 3). P1NP (formation)/CTX-1 (resorption) ratio increased $(P<0.05)$ in FTG $(127 \pm 15$ vs $150 \pm 11)$ from pre- to post-intervention, respectively, with no change in CON $(124 \pm 12$ and $123 \pm 12)$. The change in P1NP/ CTX-1 ratio was greater $(P<0.05)$ in FTG in comparison with CON.

\section{3 | Correlations}

There were no correlations between absolute or relative delta change in BMC and BMD in any body segment and the corresponding delta changes in any of the measured plasma
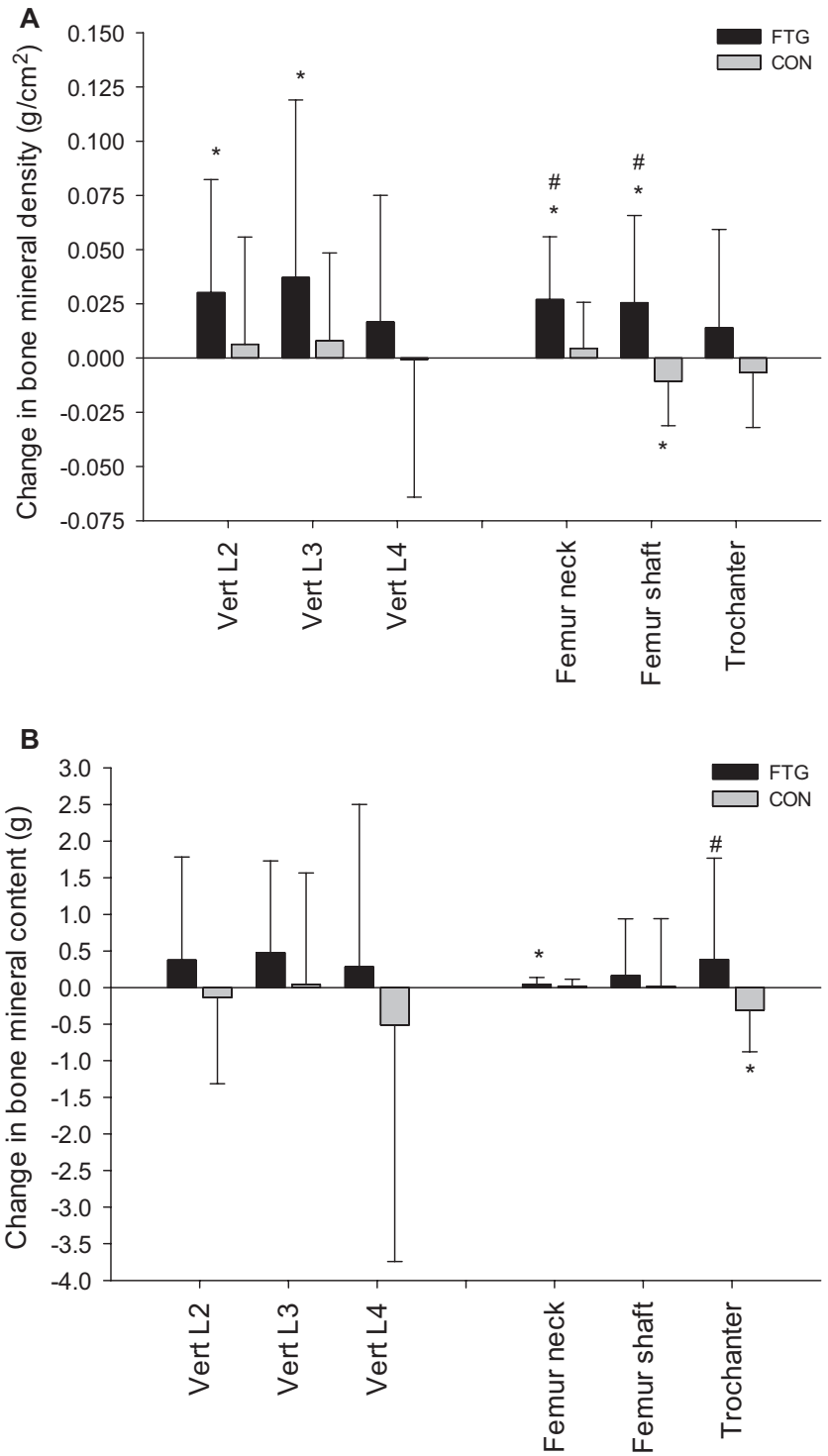

F I G URE 2 Change in bone mineral density (A) and content (B) in FTG and CON pre- and post-intervention in selected sites in the lower spine and the femur. *Denotes a significant difference from pre. \#Denotes a significant difference in change score from CON. Significant level $P<0.05$. Data are means \pm SD (standard deviation)

BTM. Nor were there any correlation between training attendance and delta change in BMC, BMD, or plasma BTM.

\section{4 | DISCUSSION}

The present study is the first RCT to investigate the osteogenic effect of recreational football training in female and male patients with prediabetes. The principal findings were that 16 weeks of recreational football training encompassing two 30-60-minute weekly sessions significantly elevated bone mineral density by $2.5 \%-3.9 \%$ in clinically important sites, such as the femur neck, trochanter, and shaft, as well as in 


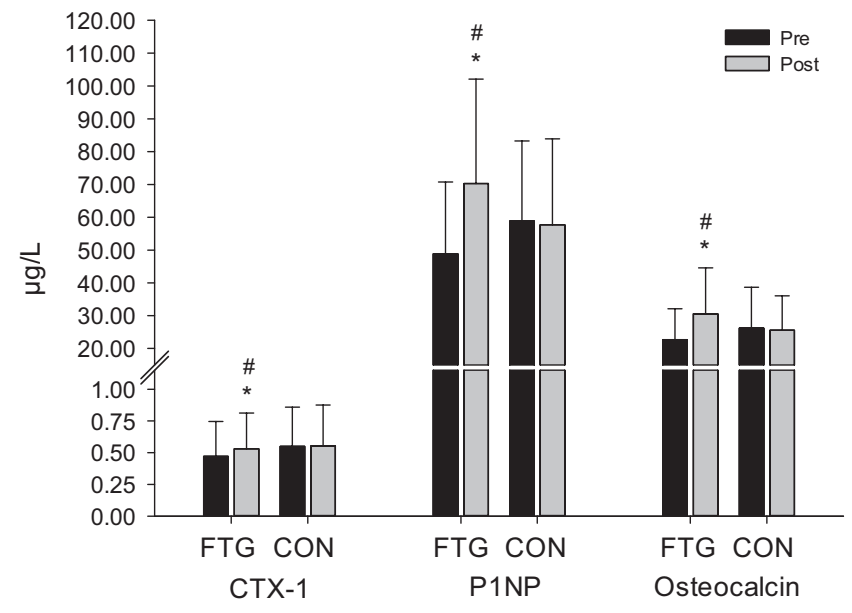

F I G URE 3 Plasma bone turnover markers in FTG and CON pre- and post-intervention. *Denotes a significant difference from pre. \#Denotes a significant difference in change score from CON. Significant level $P<0.05$. Data are means \pm SD (standard deviation)

the lower spine. In support, the football training intervention increased leg bone mineralization with $2.2 \%$ increase in bone mineral density and a $3.0 \%$ increase in bone mineral content. In line with these findings, recreational football training induced a $23 \%-52 \%$ increase in plasma bone turnover markers, representing increased osteogenesis, remodeling, and resorption.

T2DM, prediabetes, and metabolic syndrome represent pathophysiological conditions with an elevated risk of a myriad of other pathological deficiencies such as low cardiovascular and metabolic health, as well as poor bone health. ${ }^{8}$ The participants in this study had poor bone health having an average T-score of -1.6 in the femoral neck at baseline and with three of four suffering from osteoporosis or osteopenia in the femur bone (Table 1). Studies have demonstrated that bone mineralization is negatively affected by poor glycemic control, due to mechanisms not fully understood, and remains a frequently overlooked complication of prediabetes and T2DM. ${ }^{9}$ In addition, patients with deteriorated glycemic control have a higher fracture risk, independent of $\mathrm{BMD}$, compared to healthy controls. ${ }^{10,11}$ In the present study, the participants improved their blood glucose regulation and reduced body fat content markedly (see Ref. [27]), which both may have played a role in the improved bone metabolism. However, no correlations were found between bone adaptations and neither blood glucose regulation nor body fat content (data not shown).

The participants in the present study had relatively poor bone health at baseline, which may be associated with their age, sedentary lifestyle as well as poor metabolic health status. Moreover, the plasma D-vitamin levels ranged between 55 and $65 \mathrm{nmol} / \mathrm{L}$ during the intervention (see Ref. [27]), which is below the recommendations of $75-150 \mathrm{nmol} / \mathrm{L}$ for patients with deteriorated bone quality. In addition, several participants displayed levels $<50(\sim 40 \%)$ and $<75 \mathrm{nmol} / \mathrm{L}$ $(\sim 75 \%)$ (data not shown) during the experimental period. The study was conducted on the Faroe Islands during the fall and winter period, where there is limited light due to the geographical location, which is likely to have affected the low D-vitamin levels, and contributed to the poor bone health status. Plasma D-vitamin did, however, not differ between groups or pre- and post-measurements. ${ }^{27}$

Peak bone mass is attained in both genders in the second decade of life, but a decrease of approximately $1 \%$ annually occurs midlife and onwards with an accelerated decrease in women during the first few years after menopause. ${ }^{29}$ Thus, the $\sim 2 \%$ increase in leg bone mineral density and $\sim 3 \%$ increase in bone mineral content in the present study in only 16 weeks underlines that the osteogenic effects were significant. The positive adaptations (2.5\%-3.9\%) in the femoral neck and lumbar spine furthermore support the clinical importance of the present findings, as these benefits occurred in regions where a large portion of the participants had osteoporosis or osteopenia.

As evaluated by the whole-body scan, the football training group had a $3 \%$ increase in bone mineral content of the total legs corresponding to $32 \mathrm{~g}$ after 16 weeks of football training, as well as an similar percentage increase in bone mineral density. This is comparable to a $30 \mathrm{~g}$ increase in sedentary premenopausal women ${ }^{25}$ and a $41 \mathrm{~g}$ increase in 20 - to 45 -year-old untrained men ${ }^{18}$ after 15 and 12 weeks of football training, respectively. In contrast, other studies show smaller osteogenic responses ${ }^{19,24,30}$ in untrained premenopausal women, with no effects on total bone mineralization in the legs in comparable studies. ${ }^{19,31}$ Thus, the 55- to 70-yearold individuals with prediabetes in the present study display similar or even greater osteogenic adaptations than younger participants in only 16 weeks. The fact that the participants in the present study had a relatively high body weight may have reinforced the osteogenic effects of the training.

The bone loss is greater in women after the menopause than in their male counterparts with annual decreases in bone mineral density and content as high as nearly $2 \%$ and $1.5 \%$, respectively. ${ }^{32}$ However, men are additionally experiencing marked age-induced deterioration in bone health. Indeed, in the United States, around $25 \%$ of all men older than 50 years will experience a fracture due to osteoporosis. ${ }^{1}$ In the present study, the participants were representing both genders equally and the improvements in leg bone health were observed independent of gender (data not shown). Thus, football training appears to provide osteogenic stimulus and to have potential as an efficient protocol in preventing and treating osteoporosis and osteopenia in patients with relatively poor bone quality and health status.

Performing exercise training at a high intensity results in large forces being applied to bone. ${ }^{33,34}$ There appears to be consensus in the literature that the key osteogenic stimuli are 
the resulting strain magnitude and strain rate. ${ }^{13,35,36}$ Thus, exercise training with high impacts or power output ${ }^{34,37}$ is more suitable compared to training with lower intensity. Moreover, it has been suggested that complex training regimens ${ }^{16,38}$ and odd-impact sports such as team sports ${ }^{16}$ may increase the osteogenic stimuli. These types of exercise modalities may be efficient due to the diverse and intermittent forces applied to the skeleton, which may diminish the desensitization of bone that occurs with repetitive stimuli such as during continuous running. ${ }^{13,23,36}$ Indeed, several randomized controlled trials have demonstrated that recreational football can improve areal BMD and biochemical bone turnover marker profile in untrained men ${ }^{20,21,30}$ and women. ${ }^{23}$ In addition, a recent cross-sectional study demonstrated that female elite football players exhibit a 13\% higher total BMD and a $23 \%$ higher total BMC compared to untrained agematched controls. ${ }^{31}$ Thus, there appears to be support in the literature that football training provides a powerful osteogenic impact, which also applies for an elderly group with prediabetes.

In the present study plasma osteocalcin, P1NP and CTX-1 were elevated by $23 \%, 52 \%$, and $38 \%$ after 16 weeks of football training with greater increases than in the control group. This is in line with findings in football training studies both for women and men. ${ }^{20,21,30}$ These systemic markers reflect different steps in the differentiation process of the osteoblastic lineage (formation and resorption) and the marked increases in these bone markers emphasizes that football training is a potent osteogenic stimulus leading to improved bone mineralization in the legs and in the spine. Furthermore, it was observed that the P1NP (formation)/CTX-1 (resorption) ratio also increased in the football training group as result of the intervention. This indicates that the alterations in BTM induced by football training reflect an anabolic response due to an activation of the bone modeling metabolism characterized by an uncoupling of resorption (CTX-1) and formation processes (osteocalcin, P1NP), whereas bone remodeling is characterized by a site-specific coupling between resorption and formation.

In the present study, men and women took part in the football training together. This is one of the first mixed-gender RCT using football training. The findings show that football can be applied as treatment for poor bone health, and the fact that no correlation was observed between the osteogenic effect and training frequency and that both genders improved their bone health, demonstrates the high osteogenic impact and beneficial practical applications of the present treatment protocol. Finally, the broad-spectrum effects of football training ${ }^{23,39}$ implies that this exercise training method may be highly effective for patients with multiple pathophysiological conditions such as prediabetes and T2DM.

In conclusion, the present study is the first to apply football as osteogenic training for sedentary 55- to 70-year-old female and male patients with prediabetes, of which a majority suffered from femoral osteopenia or osteoporosis. The study confirms the hypothesis, that football training induces an increase in bone mineralization and a favorable change in bone turnover toward site-specific modeling, which may counteract the normal impairment of bone metabolism in this patient group.

\section{5 | PERSPECTIVES}

The present study demonstrates that 16 weeks of recreational football training causes marked beneficial bone health adaptations in 55- to 70-year-old women and men with prediabetes. Individuals suffering from prediabetes have broad-spectrum pathophysiological challenges including poor bone health. In general, the participants in the present study had poor bone health in the spine and femur and the football training improved bone mineralization at these sites as well as the overall biochemical bone turnover marker profile. Participation in small-sided football training can therefore be integrated in the treatment of prediabetic patients with poor bone health. As the bone adaptations occurred independent of gender, the study demonstrates that mixed-gender football training provides an efficient stimulus in both genders.

\section{ACKNOWLEDGEMENTS}

The authors would like to express their appreciation for the outstanding efforts and positive attitude of the participants. In addition, they are extremely grateful for the technical assistance provided by Sólfríð Skoradal, Jan Poulsen, Annika Gleðisheygg, Hjalti Gleðisheygg, Charlotta Nielsen, Brandur Jacobsen, Jens Andreassen, Johild Dulavík, Hildigunn Steinholm, Ivy Hansen, Gunnrið Jóannesarson, Ann Østerø, Nina Djurhuus, Ebba Andreassen, Maud av Fløtum, Súsanna Olsen, Synøva Hansen, Ronnie Midjord, Noomi Holm, Virgar Hvidbro, Guðrið Andorsdóttir, and Jens Jung Nielsen. The study was supported by a grant from the Faroese Research Council (Sjúkrakassagrunnurin), as well as by the Faroese Football Association (Fótbóltssamband Føroya; FSF) and the Faroese Diabetes Organisation (Diabetesfelag Føroya).

\section{ORCID}

Niklas R. Jørgensen (D) http://orcid.org/0000-0001-9624-5210

Peter Krustrup (D) http://orcid.org/0000-0002-1461-9838

Eva Wulff Helge (D) http://orcid.org/0000-0002-4351-0137

\section{REFERENCES}

1. Sidlauskas KM, Sutton EE, Biddle MA. Osteoporosis in men: epidemiology and treatment with denosumab. Clin Interv Aging. 2014;9:593-601. 
2. Rizzoli R, Bischoff-Ferrari H, Dawson-Hughes B, Weaver C. Nutrition and bone health in women after the menopause. Womens Health (Lond Engl). 2014;10:599-608.

3. National Osteoporosis Foundation. America's Bone Health: the State of Osteoporosis and Low Bone Mass in Our Nation. Washington, DC: National Osteoporosis Foundation; 2002.

4. Hernlund E, Svedbom A, Ivergård M, et al. Osteoporosis in the European Union: medical management, epidemiology and economic burden. A report prepared in collaboration with the International Osteoporosis Foundation (IOF) and the European Federation of Pharmaceutical Industry Associations (EFPIA). Arch Osteoporos 2013;8:136.

5. Gullberg B, Johnell O, Kanis JA. World-wide projections for hip fracture. Osteoporos Int. 1997;7(5):407-413.

6. Moyet J, Deschasse G, Marquant B, Mertl P, Bloch F. Which is the optimal orthogeriatric care model to prevent mortality of elderly subjects post hip fractures? A systematic review and meta-analysis based on current clinical practice. Int Orthop. 2018;[Epub ahead of print]. https://doi.org/10.1007/ s00264-018-3928-5.

7. Kohl HW, Craig CL, Lambert EV, et al. The pandemic of physical inactivity: global action for public health. Lancet. 2012;380(9838):294-305.

8. Kulkarni SV, Meenatchi S, Reeta R, Ramesh R, Srinivasan AR, Lenin C. Association of glycemic status with bone turnover markers in type 2 diabetes mellitus. Int J Appl Basic Med Res. 2017;7:247-251.

9. Starup-Linde J. Diabetes, biochemical markers of bone turnover, diabetes control, and bone. Front Endocrinol (Lausanne). 2013;4:21.

10. Nicodemus KK, Folsom AR, Study IWsH. Type 1 and type 2 diabetes and incident hip fractures in postmenopausal women. Diabetes Care. 2001;24:1192-1197.

11. Vestergaard P. Discrepancies in bone mineral density and fracture risk in patients with type 1 and type 2 diabetes - a meta-analysis. Osteoporosis. 2007;18:427-444.

12. Turner $\mathrm{CH}$. Homeostatic control of bone-structure-an application of feedback theory. Bone. 1991;12:203-217.

13. Turner $\mathrm{CH}$, Robling AG. Designing exercise regimens to increase bone strength. Exerc Sport Sci Rev. 2003;31:45-50.

14. Lanyon LE. Using functional loading to influence bone mass and architecture: objectives, mechanisms, and relationship with estrogen of the mechanically adaptive process in bone. Bone. 1996;18:S37-S43.

15. Zhao M, Zhang L. Efficiency of jumping exercise in improving bone mineral density among premenopausal women: a metaanalysis. Sports Med. 2014;44:1393-1402.

16. Nikander R, Kannus P, Dastidar P, Hannula M, Harrison L, Cervinka $\mathrm{T}$, et al. Targeted exercises against hip fragility. Osteoporos Int. 2009;20:1321-1328.

17. Beck BR, Daly RM, Singh MA, Taaffe DR. Exercise and Sports Science Australia (ESSA) position statement on exercise prescription for the prevention and management of osteoporosis. $J$ Sci Med Sport. 2017;20:438-445.

18. Krustrup P, Nielsen JJ, Krustrup BR, et al. Recreational soccer is an effective health-promoting activity for untrained med. $\mathrm{Br} \mathrm{J}$ Sports Med. 2009;43(11):825-831.

19. Krustrup P, Hansen PR, Randers MB, et al. Beneficial effects of recreational football on the cardiovascular risk profile in untrained premenopausal women. Scand J Med Sci Sports. 2010a;20(Suppl. 1):40-49.

20. Helge EW, Randers MB, Hornstrup T, et al. Street football is a feasible health-enhancing activity for homeless men - bone marker profile and balance improved. Scand J Med Sci Sports. 2014;24(Suppl 1):122-129.

21. Uth J, Hornstrup T, Christensen JF, et al. Efficacy of recreational football on bone health, body composition, and physical functioning in men with prostate cancer undergoing androgen deprivation therapy: 32-week follow-up of the FC prostate randomised controlled trial. Osteoporosis Int. 2016;27:1507-1518.

22. Randers MB, Petersen J, Andersen LJ, et al. Short term street soccer improves fitness and cardiovascular health status of homeless men. Eur J Appl Physiol. 2012;112:2097-2106.

23. Krustrup P, Helge EW, Hansen PR, et al. Effects of recreational football on women's fitness and health: adaptations and mechanisms. Eur J Appl Physiol. 2018;118:11-32.

24. Helge EW, Aagaard P, Jakobsen MD, et al. Recreational football training decreases risk factors for bone fractures in untrained premenopausal women. Scand J Med Sci Sports. 2010;20(Suppl 1):31-39.

25. Mohr M, Helge EW, Petersen LF, et al. Effects of soccer vs swim training on bone formation in sedentary middle-aged women. Eur J Appl Physiol. 2015;115:2671-2679.

26. Veyhe AS, Andreassen J, Halling J, Grandjean P, Petersen MS, Weihe P. Prevalence of type 2 diabetes and prediabetes in the Faroe Islands. Diabetes Res Clin Pract. 2018;140:162-173.

27. Skoradal MB, Weihe $\mathrm{P}$, Patursson $\mathrm{P}$, et al. Football training improves metabolic and cardiovascular health status in 55- to 70-year-old women and men with prediabetes. Scand J Med Sci Sports. 2018;28(Suppl. 1):42-51.

28. Randers MB, Nybo L, Petersen J, et al. Activity profile and physiological response to football training for untrained males and females, elderly and youngsters: influence of the number of players. Scand J Med Sci Sports. 2010;20(Suppl. 1):14-23.

29. Bonjour JP, Chevalley T, Ferrari S, Rizzoli R. The importance and relevance of peak bone mass in the prevalence of osteoporosis. Salud Publica Mex. 2009;51(Suppl 1):5-17.

30. Helge EW, Andersen TR, Schmidt JF, et al. Recreational football improves bone mineral density and bone turnover marker profile in elderly men. Scand J Med Sci Sports. 2014;24(Suppl 1):98-104

31. Jackman SR, Scott S, Randers MB, et al. Musculoskeletal health profile for elite female footballers versus untrained young women before and after 16 weeks of football training. J Sports Sci. 2013;31:1468-1474.

32. Ahlborg HG, Johnell O, Turner $\mathrm{CH}$, Rannevik G, Karlsson MK. Bone loss and bone size after menopause. $N$ Engl J Med. 2003;349(4):327-334.

33. Bennell KL, Malcolm SA, Khan KM, et al. Bone mass and bone turnover in power athletes, endurance athletes, and controls: a 12 month longitudinal study. Bone. 1997;20:477-484.

34. von Stengel S, Kemmler W, Kalender WA, Engelke K, Lauber D. Differential effects of strength versus power training on bone mineral density in postmenopausal women: a 2-year longitudinal study. Br J Sports Med. 2007;41:649-655.

35. Lanyon LE. Using functional loading to influence bone mass and architecture: objectives, mechanisms, and relationship with 
estrogen of the mechanically adaptive process in bone. Bone. 1996;18(Suppl 1):37-43.

36. Turner $\mathrm{CH}$. Three rules for bone adaptation to mechanical stimuli. Bone. 1998;23(Suppl 5):399-407.

37. Gianoudis J, Bailey CA, Ebeling PR, et al. Effects of a targeted multimodal exercise program incorporating high-speed power training on falls and fracture risk factors in older adults: a community-based randomized controlled trial. J Bone Miner Res. 2014;29(1):182-191.

38. Vincent KR, Braith RW. Resistance exercise and bone turnover in elderly men and women. Med Sci Sports Exerc. 2002;34:17-23.

39. Milanović Z, Pantelić S, Čović N, Sporiš G, Mohr M, Krustrup P. Broad-spectrum physical fitness benefits of recreational football: a systematic review and meta-analysis. $\mathrm{Br} J$ Sports Med. 2018. [Epub ahead of print] https://doi.org/10.1136/ bjsports-2017-097885

How to cite this article: Skoradal M-B, Helge EW, Jørgensen NR, et al. Osteogenic impact of football training in 55- to 70-year-old women and men with prediabetes. Scand J Med Sci Sports. 2018;28(Suppl. 1): 52-60. https://doi.org/10.1111/sms.13252 